\title{
Universiteit
}

Leiden

The Netherlands

\section{Charge dynamics of doped holes in high Tc cuprate superconductors: A clue from optical conductivity}

Mishchenko, A.S.; Nagaosa, N.; Shen, Z.X.; Filippis, G. de; Cataudella, V.; Devereaux, T.P.; ... ; Zaanen, J.

\section{Citation}

Mishchenko, A. S., Nagaosa, N., Shen, Z. X., Filippis, G. de, Cataudella, V., Devereaux, T. P., ... Zaanen, J. (2008). Charge dynamics of doped holes in high Tc cuprate superconductors: A clue from optical conductivity. Physical Review Letters, 100(16), 166401.

doi:10.1103/PhysRevLett.100.166401

Version: $\quad$ Not Applicable (or Unknown)

License: $\quad$ Leiden University Non-exclusive license

Downloaded from: https://hdl.handle.net/1887/62685

Note: To cite this publication please use the final published version (if applicable). 


\title{
Charge Dynamics of Doped Holes in High $T_{c}$ Cuprate Superconductors: A Clue from Optical Conductivity
}

\author{
A. S. Mishchenko, ${ }^{1,2}$ N. Nagaosa, ${ }^{1,3}$ Z.-X. Shen, ${ }^{4}$ G. De Filippis, ${ }^{5}$ V. Cataudella, ${ }^{5}$ T. P. Devereaux,${ }^{6,4}$ C. Bernhard, ${ }^{7}$ \\ K. W. Kim, ${ }^{7}$ and J. Zaanen ${ }^{8}$ \\ ${ }^{1}$ Cross-Correlated Materials Research Group (CMRG), ASI, RIKEN, Wako 351-0198, Japan \\ ${ }^{2}$ RRC “Kurchatov Institute," 123182, Moscow, Russia \\ ${ }^{3}$ Department of Applied Physics, The University of Tokyo, 7-3-1 Hongo, Bunkyo-ku, Tokyo 113, Japan \\ ${ }^{4}$ Department of Physics, Applied Physics, and Stanford Synchrotron Radiation Laboratory, Stanford University, \\ Stanford, California 94305, USA \\ ${ }^{5}$ Coherentia-CNR-INFM and Dipartamento di Scienze Fisiche-Università di Napoli Federico II-I-80126 Napoli, Italy \\ ${ }^{6}$ Department of Physics, University of Waterloo, Ontario, Canada N2L 3GI \\ ${ }^{7}$ Department of Physics and Fribourg Center for Nanomaterials, University of Fribourg, CH-1700 Fribourg, Switzerland \\ ${ }^{8}$ Instituut Lorentz for Theoretical Physics, Leiden University, POB 9506, 2300 RA Leiden, The Netherlands
}

(Received 7 December 2007; published 24 April 2008)

\begin{abstract}
The charge dynamics in weakly hole doped high temperature superconductors is studied in terms of the accurate numerical solution to a model of a single hole interacting with a quantum lattice in an antiferromagnetic background, and accurate far-infrared ellipsometry measurements. The experimentally observed two electronic bands in the infrared spectrum can be identified in terms of the interplay between the electron correlation and electron-phonon interaction resolving the long standing mystery of the midinfrared band.
\end{abstract}

DOI: 10.1103/PhysRevLett.100.166401

It is now widely recognized that the physics of doping holes into a Mott insulator is the key concept to understand the high temperature superconductivity in cuprates [1]. An appealing scenario is that the spin singlet pairs already existing in the insulating antiferromagnet turn into the superconducting Cooper pairs when the doped holes introduce the charge degrees of freedom. On the other hand, it is also noted that the parent compound is an ionic insulator, where the polar electron-phonon interaction (EPI) plays an essential role, and it is expected that this strong EPI continues to be of quite vital importance even at finite doping. Therefore, the quantum dynamics of the doped holes is essentially influenced by both the magnetic fluctuations and quantum phonons.

It has been recognized that the charge dynamics is determined not by the large Fermi surface but by the doped holes in the underdoped region [1]. Therefore, it is a reasonable approach to consider the charge-current dynamics of the holes (not electrons) interacting with the quantum phonons and magnons simultaneously to analyze the infrared optical conductivity (OC). The basic features of the observed d.c. conductivity and OC follow Refs. [24]. The OC $\sigma(\omega)$ in undoped material reveals the charge transfer band at $\omega \cong 1.5 \mathrm{eV}$ between the $p$ orbitals of oxygen and $d$ orbitals of copper. With doping, a low energy part of $\sigma(\omega)$ develops revealing the dynamics of the doped holes as a function of the frequency $\omega$. In particular, the Drude weight is shown to be proportional to $x$ even in the absence of antiferromagnetic long range order, while the relaxation rate $1 / \tau$ is proportional to the temperature $T$. Therefore, this dependence of the Drude weight finds a
PACS numbers: 74.72. $-\mathrm{h}, 71.10 . \mathrm{Fd}, 71.38 .-\mathrm{k}, 75.50 . \mathrm{Ee}$

natural explanation as directly reflecting the hole concentration, as mentioned above, while the $T$ dependence is due to the lifetime of the holes. The higher frequency $\sigma(\omega)$, on the other hand, has not been well understood. Especially the midinfrared (MIR) peak with dependent on doping energy at around $\omega_{\mathrm{MIR}} \cong 0.5 \mathrm{eV}$ is still controversial [5], with interpretations involving an $\omega$ dependence of $1 / \tau$, transitions between the Zhang-Rice singlet state to the upper Hubbard band, and magnon sidebands.

For this problem, angle-resolved-photoemission spectroscopy (ARPES) offers an important clue. ARPES in undoped parent compounds measures the spectral function of a single hole left behind when an electron is kicked out from the sample by the incident light [6,7]. The corresponding theoretical analysis has pointed out the role of the composite polaronic effect due to electron-magnon and electron-phonon $(e-\mathrm{ph})$ couplings $[8,9]$. Therefore, it is expected that these two interactions are indispensable to the understanding of infrared optical spectra as well.

We study in this Letter theoretically the OC of the single-hole doped into a Mott insulator described by the $t-J$-Holstein model, and its numerical solution in terms of the diagrammatic Monte Carlo (DMC) simulation. Previously, the OC of the $t$ - $J$-Holstein model has been calculated by exact diagonalization of small clusters [10], in the noncrossing approximation (NCA) for both magnetic and lattice variables [11], and for the case of infinite dimension [12]. Compared with these approximate methods, our DMC simulation provides more accurate solution for the infinite system without approximation associated with the phonon sector [13] and for proper lattice geometry 
and dimension. The only approximation is the NCA for magnons which is shown to be sufficiently good for the parameters considered below $[14,15]$. These results are compared with accurate far-infrared ellipsometry measurement as well as with previously published data. The infrared ellipsometry measurements have been performed with a home-built ellipsometer attached to a Bruker fast-Fourier spectrometer at the IR-beam line of the ANKA synchrotron at FZ Karlsruhe, D at $70-700 \mathrm{~cm}^{-1}$ and with conventional light sources at $500-7000 \mathrm{~cm}^{-1}[16]$.

Figure 1 summarizes our main results, where four panels for the infrared parts of $\sigma(\omega)$ are compared. Figures 1(a)1(c) show the calculated $\sigma(\omega)$ for the Holstein model ( $e$-ph coupling only), $t-J$ model (el-magnon only), and the $t$ - $J$-Holstein model ( $e$-ph and el-magnon couplings), respectively, while Fig. 1(d) presents the experimental observation. Neither the $t-J$ model 1(b), nor the Holstein model 1(a) bear a resemblance to the experiment 1(d), while their combination 1(c) at least qualitatively reproduces the salient experimental features. The clear signature of the experiment is that there are two prominent electronic components, i.e., the so-called MIR band at around $\omega_{\text {MIR }}=4600 \mathrm{~cm}^{-1}$ and the lower energy one at around $\omega=1000 \mathrm{~cm}^{-1}$ that is located just above the infrared active phonon modes which show up as sharp peaks below $800 \mathrm{~cm}^{-1}$. The lower energy peak roughly corresponds to that seen in Fig. 1(c) due to the phonon sideband. However,
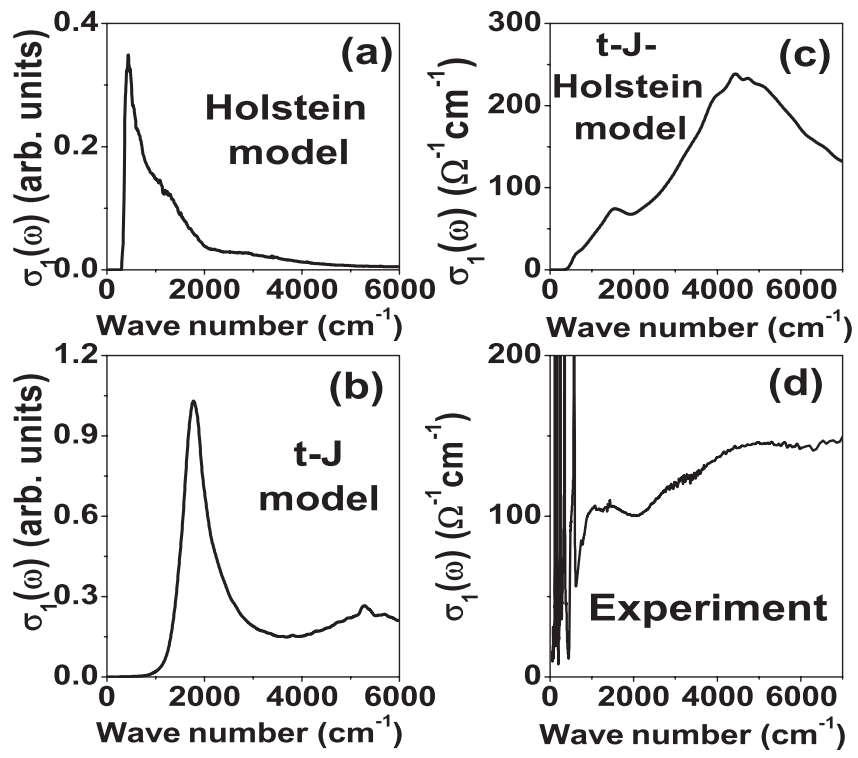

FIG. 1. Comparison of typical OCs of different models in 2D with experimental data of heavily underdoped cuprates: (a) Holstein model at $\lambda=0.44$; (b) $t$ - $J$ model at $J=0.3$; (c) $t$ - $J$-Holstein model for $J=0.3$ and $\lambda=0.39$; (d) in-plane OC of $1.5 \%$ hole doped $\left(\mathrm{Eu}_{1-x} \mathrm{Ca}_{x}\right) \mathrm{Ba}_{2} \mathrm{Cu}_{3} \mathrm{O}_{6}$ at $T=10 \mathrm{~K}$. The energy dependence of the theoretical data is presented in wave numbers assuming $t=0.3 \mathrm{eV}\left(1 \mathrm{eV}=8065.5 \mathrm{~cm}^{-1}\right)$. The absolute value of the theoretical $\sigma_{1}$ is evaluated using the experimental hopping distance $a=3.86 \AA$ and bulk hole concentration $n_{h}=1.72 \times 10^{-23} \mathrm{~cm}^{-3}$. the MIR peak does not correspond to that of the $t-J$ model which occurs at around $\omega_{t-J} \cong 2 J \cong 2000 \mathrm{~cm}^{-1}$. Instead of that, according with experiment, it is shifted to higher energies.

As it can be seen from the results below, the coupling to two kinds of bosonic excitations results in two separate peaks because of the essentially different nature of the electron-magnon and $e$-ph couplings [17] and significantly different energy scales of the magnetic and lattice excitations which are involved in the optical transitions. The magnons with large characteristic energy $\sim 2 J$ are weakly bound to the hole. To the contrary, the phonons are adiabatic and the EPI is considerable. As shown below, the lower energy peak is the phonon sideband with the threshold at the phonon energy [18] and the higher energy peak is the magnon sideband of the lower peak. The reason for the apparent shift of the $2 J$ peak to higher energies is most evident in the strong-coupling limit where the FranckCondon picture for optical processes is valid [18] and the fluctuations of energies of different lattice sites, with the characteristic scale of Franck-Condon energy, can be considered as being frozen. Then, the energy cost of the transition of the hole from the ground state to excited states of the $t-J$ model with frozen lattice is the sum of the energy of the emitted magnon and the Franck-Condon energy. Hence, the two peaks in the OC are the consequence of the importance of both $e$-ph and magnetic interactions. The same information is encoded in a different way in the single-particle spectral function observed in ARPES, where the low energy quasiparticle peak of $t-J$ model is affected by $e$-ph interaction. This low energy peak is separated into the broad Franck-Condon peak mimicking the dispersion of the $t-J$ model while the zero-phonon line with very small weight has almost no dispersion $[7,8]$.

In the standard spin-wave approximation for the $t-J$ model $[14,19]$, the dispersionless hole $\varepsilon_{0}=$ const (annihilation operator is $h_{\mathbf{k}}$ ) propagates in the magnon (annihilation operator is $\alpha_{\mathbf{k}}$ ) bath

$$
\hat{H}_{t-J}^{0}=\sum_{\mathbf{k}} \varepsilon_{0} h_{\mathbf{k}}^{\dagger} h_{\mathbf{k}}+\sum_{\mathbf{k}} \omega_{\mathbf{k}} \alpha_{\mathbf{k}}^{\dagger} \alpha_{\mathbf{k}}
$$

with magnon dispersion $\omega_{\mathbf{k}}=2 J \sqrt{1-\gamma_{\mathbf{k}}^{2}}$, where $\gamma_{\mathbf{k}}=$ $\left(\cos k_{x}+\cos k_{y}\right) / 2$. The hole is scattered by magnons

$$
\hat{H}_{t-J}^{h-m}=N^{-1 / 2} \sum_{\mathbf{k}, \mathbf{q}} M_{\mathbf{k}, \mathbf{q}}\left[h_{\mathbf{k}}^{\dagger} h_{\mathbf{k}-\mathbf{q}} \alpha_{\mathbf{k}}+\text { H.c. }\right]
$$

with the standard scattering vertex $M_{\mathbf{k}, \mathbf{q}}$ [4].

The OC of the $t-J$ model has been calculated by various methods in numerous papers giving mutually consistent results, e.g., [20,21]. To confirm the validity of our method, we first reproduce the results for different $J / t$ ratios [22]. We find the well-known peak at around $\omega_{t-J} \cong 2 J$, the origin of which has not been settled yet. It is also difficult to identify this peak with the observed MIR peak since the 


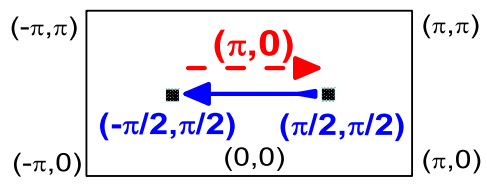

FIG. 2 (color online). Electronic transition (solid arrow) and emitted magnon (dashed arrow) responsible for the $2 J$ peak in the OC of the $t$ - $J$ model.

energy of the latter is about 2 times higher than $2 J$. Another source for skepticism is the opposite doping dependence of the $\omega_{t-J}$ and $\omega_{\text {MIR }}$ energies [21]. Therefore, we conclude that the $t-J$ model cannot explain the observed OC even at very small dopings. Before introducing the EPI, let us first provide an interpretation of the $\omega_{t-J} \cong 2 J$ peak. This peak comes from the hole excitations within the coherent band of the $t$ - $J$ model from the ground state at $(\pi / 2, \pi / 2)$ to the neighborhood of $(-\pi / 2, \pi / 2)$ point, assisted by emission of single magnon with energy $\approx 2 J$ and a momentum $(\pi, 0)$ (Fig. 2). To prove this point we computed the OC by disentangling magnon and hole terms (exact at the lowest order in $t$ ) and then calculating the correlation function involving the hole operators in the subspace with one magnon (see upper panel in Fig. 3)

$$
\begin{aligned}
\Re \sigma(\omega)= & 4 \pi t^{2} e^{2}(\omega N)^{-1} \sum_{\vec{q}}\left|\left\langle\psi_{\vec{k}_{0}-\vec{q}}^{(1)}\left|O_{\vec{q}}\right| \psi_{\vec{k}_{0}}^{(1)}\right\rangle\right|^{2} \\
& \times \delta\left[\omega-\omega_{q}-\left(E_{\vec{k}_{0}-\vec{q}}^{(1)}-E_{\vec{k}_{0}}^{(1)}\right)\right] .
\end{aligned}
$$

Here $\left|\psi_{\vec{k}}^{(1)}\right\rangle$ is the lowest eigenstate in the subspace with one magnon with energy $E_{\vec{k}}^{(1)}, \vec{k}_{0}=(\pi / 2, \pi / 2)$, and $O_{\vec{q}}=$ $\sum_{\vec{k}} h_{\vec{k}-\vec{q}}^{\dagger} h_{\vec{k}} C(\vec{k}-\vec{q}, \vec{k})$ [23]. By direct inspection of the sum over $\mathbf{q}$ in Eq. (3) we found that the main contribution to the OC comes from the transfer of magnon momentums

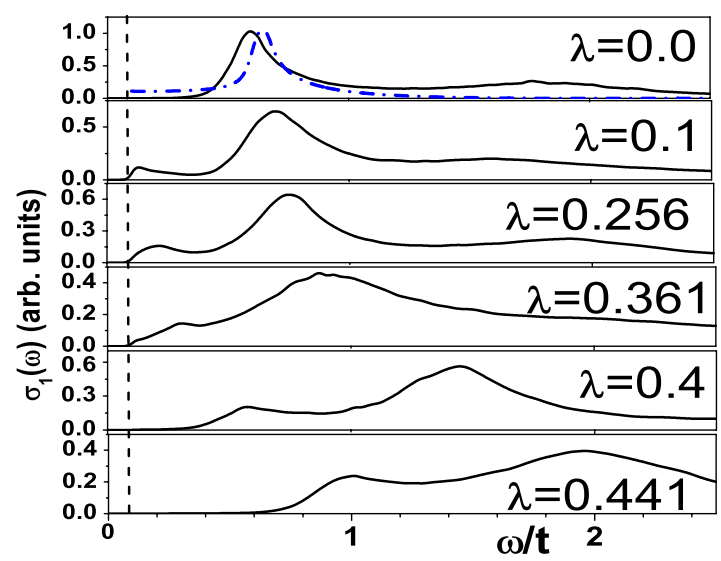

FIG. 3 (color online). OC of a single hole in the $t$ - $J$-Holstein model at $J / t=0.3$ with various EPI coupling constants $\lambda$. The vertical dashed line at $\omega / t=0.1$ indicates the phonon frequency. The dash-dotted line in the upper panel is the result of Eq. (3). around $(\pi, 0)$. It can be traced out from the $\mathbf{q}$ dependence of the vertex $\left|C\left(\mathbf{k}_{0}-\mathbf{q}, \mathbf{k}_{0}\right)\right|^{2}$.

We now turn to the EPI added to the $t-J$ model, the importance of which has been already established as mentioned above. When the model is updated to the $t-J$-Holstein model, the hole interacts with dispersionless (frequency $\Omega=$ const) optical phonons by short range coupling $\gamma$

$$
\hat{H}^{e-\mathrm{ph}}=\Omega \sum_{\mathbf{k}} b_{\mathbf{k}}^{\dagger} b_{\mathbf{k}}+\frac{\gamma}{\sqrt{N}} \sum_{\mathbf{k}, \mathbf{q}}\left[h_{\mathbf{k}}^{\dagger} h_{\mathbf{k}-\mathbf{q}} b_{\mathbf{k}}+\text { H.c. }\right] .
$$

In units of $t=1$ we parametrize the dimensionless EPI constant as $\lambda=\gamma^{2} /(4 t \Omega)$, choosing the value $J / t=0.3$, and setting the phonon frequency $\Omega=0.1$ [24].

Figure 3 shows the effect of EPI on the OC of a single hole in the $t$ - $J$-Holstein model. At weak EPI, an absorption starts and shows a peak right above the phonon frequency. This apparent two-peak structure of the MIR response of underdoped cuprates can be tacitly discerned from many previous measurements (Fig. 3 in [25] and Fig. 9 in [2]) and is clearly seen from the low-temperature in-plane OC of $1.5 \%$ hole doped $\mathrm{Eu}_{1-x} \mathrm{Ca}_{x} \mathrm{Ba}_{2} \mathrm{Cu}_{3} \mathrm{O}_{6}$ measured by ellipsometry [Fig. 1(d)]. This low energy EPImediated peak stays close to phonon energy up to the selftrapping transition point which is, for given parameters of the model, located at $\lambda \approx 0.4$ [8]. Indeed, according to the dependence of the dominant $2 J$ contribution and the low energy peak on $\lambda$ [Fig. 4(a)], the transition from the weakto the strong-coupling regime occurs at this coupling strength. We note that both the $t-J$ model and polaron
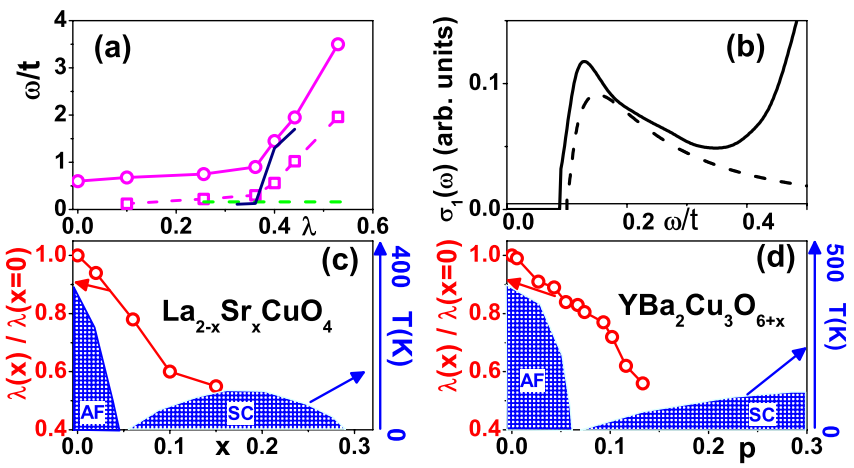

FIG. 4 (color online). (a) EPI coupling dependence of the energies of the dominating peak (solid line with circles) and EPI-mediated feature (dashed line with squares) of the OC. The energies of dominating phonon peak of the $\mathrm{OC}$ of the Holstein model at $t=1$ (dashed line) and those at $\tilde{t}=0.4$ (solid line). (b) OC of the $t$ - $J$-Holstein model (solid line) and OC of the effective Holstein model with $\tilde{t}=0.4$ (dashed line) at the same coupling $\lambda=0.1$. Ratio of the effective EPI constant at doping $x$ (or real in-plane concentration $p$ for YBCO [31]) to that at zero doping estimated from the MIR peak position for (c) LSCO and (d) YBCO mapped on the phase diagrams. 
physics is crucial to explain the very existence of the twopeak structure of the OC.

To understand the nature of the low energy peak induced by EPI, we did a calculation of OC for the Holstein model without hole-magnon interaction with reduced transfer $\tilde{t}=0.4 t$ mimicking the mass enhancement, which reproduces the self-trapping point of $t-J$-Holstein model with $t=1$. As seen in Fig. 4(b), this effective Holstein model reproduces remarkably well the shape of the low energy feature of OC for the $t-J$-Holstein model.

Since the effective EPI decreases with doping $[26,27]$ the reason of the experimentally observed $[5,28,29]$ MIR mode softening is the change of the EPI. Comparing the position of the MIR mode with results of the $t$ - $J$-Holstein model we can give a rough estimate of the renormalization of the effective EPI with doping. First, since the selftrapping point of the realistic extended $t t^{\prime} t^{\prime \prime}-J$ model is $\lambda_{\text {st }}^{t t^{\prime} t^{\prime}-J} \approx 0.6$ [30], we scale the EPI strength in the Fig. 4(a) as $\lambda \rightarrow 1.5 \lambda$. Second, since the quadratic dependence of the energy scales of the OC response on $\lambda$ is a known property of the strong coupling regime [18], we extrapolate the OC data to larger EPI couplings. Using a quadratic extrapolation of the experimental data of the MIR peak in $\mathrm{YBa}_{2} \mathrm{Cu}_{3} \mathrm{O}_{6+x}$ (YBCO) [5,28] and $\mathrm{La}_{2-x} \mathrm{Sr}_{x} \mathrm{CuO}_{4}$ (LSCO) [29] to zero dopings, we arrive at the result in Figs. 4(c) and 4(d) which is in agreement with [26]. Moreover, since the analysis of the ARPES in undoped LSCO gives $\lambda \sim 1$ [9], the data in Figs. 4(c) and 4(d) give absolute values of $\lambda^{\mathrm{LSCO}}(x)$. Finally, our result for $\lambda^{\mathrm{LSCO}}(x)$ is in quantitative agreement with the values obtained from the "kink" angle in ARPES on LSCO [27]. Figures 4(c) and 4(d) strongly suggest that superconductivity appears after the effective EPI decreases from strong to weak coupling, which liberates the coherent motion of the doped holes. The $x$ axis for YBCO should be translated to the hole doping concentration in the $\mathrm{CuO}_{2}$ planes $p$, which makes the two phase diagrams, Figs. 4(c) and 4(d), look almost similar. Therefore, the behavior of the effective EPI and phase diagram seem almost universal in high- $T_{c}$ cuprates. However, the discussion above is restricted to the polaronic effect for the holes and not for the quasiparticles forming the large Fermi surface. The contribution of the EPI to the pairing hence is not excluded by the present analysis.

Fruitful discussions with N. V. Prokof'ev, B. V. Svistunov, and G.A. Sawatzky are acknowledged. A. S. M. is supported by RFBR No. 07-02-00067a. N. N. was partly supported by the Grant-in-Aids from under the Grant No. 15104006, No. 16076205, No. 17105002, and No. 19048015, and NAREGI Nanoscience Project from the Ministry of Education, Culture, Sports, Science, and Technology, Japan. C. B. and K. W. K. acknowledge technical support of Y.L. Mathias at the ANKA IR-beam line and funding by the Schweizer Nationalfonds (SNF) through Grant No. 200021-111690 and the Deutsche Forschungsgemeinschaft (DFG) through Grant No. BE 2684/1 in FOR538.
[1] P. A. Lee, N. Nagaosa, and X.-G. Wen, Rev. Mod. Phys. 78, 17 (2006).

[2] D. N. Basov and T. Timusk, Rev. Mod. Phys. 77, 721 (2005).

[3] M. A. Kastner et al., Rev. Mod. Phys. 70, 897 (1998).

[4] E. Manousakis, Rev. Mod. Phys. 63, 1 (1991); E. Dagotto, ibid. 66, 763 (1994).

[5] Y. S. Lee et al., Phys. Rev. B 72, 054529 (2005).

[6] A. Damascelli, Z.-X. Shen, and Z. Hussain, Rev. Mod. Phys. 75, 473 (2003).

[7] K. M. Shen et al., Phys. Rev. Lett. 93, 267002 (2004).

[8] A.S. Mishchenko and N. Nagaosa, Phys. Rev. Lett. 93, 036402 (2004).

[9] O. Rösch et al., Phys. Rev. Lett. 95, 227002 (2005).

[10] B. Baüml, G. Wellein, and H. Fehske, Phys. Rev. B 58, 3663 (1998).

[11] B. Kyung et al., Phys. Rev. B 54, 13167 (1996).

[12] E. Cappelluti, S. Ciuchi, and S. Fratini, Phys. Rev. B 76, 125111 (2007).

[13] A. S. Mishchenko et al., Phys. Rev. B 62, 6317 (2000); A. S. Mishchenko and N. Nagaosa, J. Phys. Soc. Jpn. 75, 011003 (2006).

[14] Z. Liu and E. Manousakis, Phys. Rev. B 45, 2425 (1992).

[15] O. Gunnarsson and O. Rösch, Phys. Rev. B 73, 174521 (2006).

[16] C. Bernhard, J. Humlicek, and B. Keimer, Thin Solid Films 455-456, 143 (2004).

[17] V. Cataudella et al., Phys. Rev. Lett. 99, 226402 (2007).

[18] A.S. Mishchenko et al., Phys. Rev. Lett. 91, 236401 (2003); G. De Filippis et al., ibid. 96, 136405 (2006).

[19] C. L. Kane, P. A. Lee, and N. Read, Phys. Rev. B 39, 6880 (1989).

[20] T. M. Rice and F. C. Zhang, Phys. Rev. B 39, 815 (1989); W. Stephan and P. Horsch, ibid. 42, 8736 (1990); D. Poilblanc and E. Dagotto, ibid. 44, 466 (1991); D. Poilblanc, ibid. 44, 9562 (1991); E. Dagotto et al., ibid. 45, 10741 (1992); J. I. Igarashi and P. Fulde, ibid. 48, 12713 (1993); J. Jaklič and P. Prelovšek, ibid. 50, 7129 (1994); G. Jackeli and N. M. Plakida, ibid. 60, 5266 (1999); M. Moraghebi, S. Yunoki and A. Moreo, ibid. 66, 214522 (2002); B. Kyung and S. I. Mukhin, ibid. 55, 3886 (1997); N. M. Plakida, Z. Phys. B 103, 383 (1997).

[21] R. Eder, P. Wróbel, and Y. Ohta, Phys. Rev. B 54, R11 034 (1996).

[22] Since the current operator is not diagonal in the momentum space, we need some further technical development for the calculation of the OC [18]: A. S. Mishchenko et al. (to be published).

[23] The vortex $C(\vec{k}-\vec{q}, \vec{k})$ is defined in [11].

[24] In high $T_{c}$ materials $t \approx 0.3-0.43 \mathrm{eV}$ [4], $\Omega \approx$ 55-80 meV [6].

[25] G. A. Thomas et al., Phys. Rev. B 45, 2474 (1992).

[26] A. Lanzara et al., Nature (London) 412, 510 (2001).

[27] A. S. Mishchenko et al. (unpublished).

[28] S. L. Cooper et al., Phys. Rev. B 47, 8233 (1993).

[29] S. Uchida et al., Phys. Rev. B 43, 7942 (1991).

[30] A.S. Mishchenko and N. Nagaosa, Phys. Rev. B 73, 092502 (2006).

[31] R. Liang, D. A. Bonn and W. N. Hardy, Phys. Rev. B 73, 180505(R) (2006). 